\title{
Models of semiconductor quantum dots blinking based on the spectral diffusion
}

\author{
Vladislav Busov and Pavel Frantsuzov* \\ Lomonosov Moscow State University, 119991 Moscow, Russia
}

Two decades have passed since the first observation of long-term fluorescence intensity fluctuations (blinking) of single colloidal CdSe quantum dots (QDs) with a $\mathrm{ZnS}$ shell [1]. In further experimental studies it was found (see [2-4]) that these fluctuations have a wide spectrum of characteristic timescales, from hundreds of microseconds to hours.

Another interesting phenomenon manifested in the emission of single quantum dots is spectral diffusion showing the characteristic time scales in order of hundreds of seconds [56]. It is not surprising that there are a number of models connecting the fluctuations of the emission intensity with the slow motion of the exciton energy. The first model of that kind suggested by Shimizu et al. [7] is based on the Efros/Rosen charging mechanism [8]. More advanced version of this idea was suggested by Tang and Marcus [9-10] as the diffusioncontrolled electron transfer model (DCET). In 2014 Zhu and Marcus [11] presented an extension of the DCET model by introduction of an additional biexciton charging channel. Simultaneously with the Tang and Marcus, the model based on the alternative fluctuating rate mechanism of blinking was suggested by Frantsuzov and Marcus [12]. The mechanism assumes that the variations of the non-radiative exciton recombination rate $k_{n}$ generate fluctuations of the emission intensity on a long time scale. The Frantsuzov and Marcus model connects the recombination rate with the fluctuating energy difference between $1 \mathrm{~S}$ and $1 \mathrm{P}$ states.

The predictions of the DCET model [9-10], the Expend DCET model [11], and the Frantsuzov and Marcus model [12] were compared by the analytical analysis and numerical simulations. It was found that the DCET model [9-10] predicts the appearance of extremely long ON and OFF time periods in a single QD emission trace. As seen on Fig.1 such a period could last years, which is much longer than the duration of a typical experiment. Thus the QD can become permanently bright or permanently dark after about a hundred blinking cycles with a high probability. The presence of a second ionization channel in the Extended DCET [11] model resolves the problem with very long ON times, but not with the very long OFF times. As a result, the most of the QD has to become permanently dark in this model as confirmed by numerical simulations. These predictions significantly differ from the experimentally observed behaviour of single quantum dots. On the contrary, the predictions of the model of Frantsuzov and Marcus [12] correspond to the basic properties of the quantum dots blinking obtained in experiment, such as a nearly continuous set of emission intensities,

\footnotetext{
* Corresponding author: pavel.frantsuzov@gmail.com
} 
the power-law form of the ON-time and OFF-time distributions obtained by the threshold procedure, and a change in behavior the of the emission intensity power spectral density on millisecond time scales.

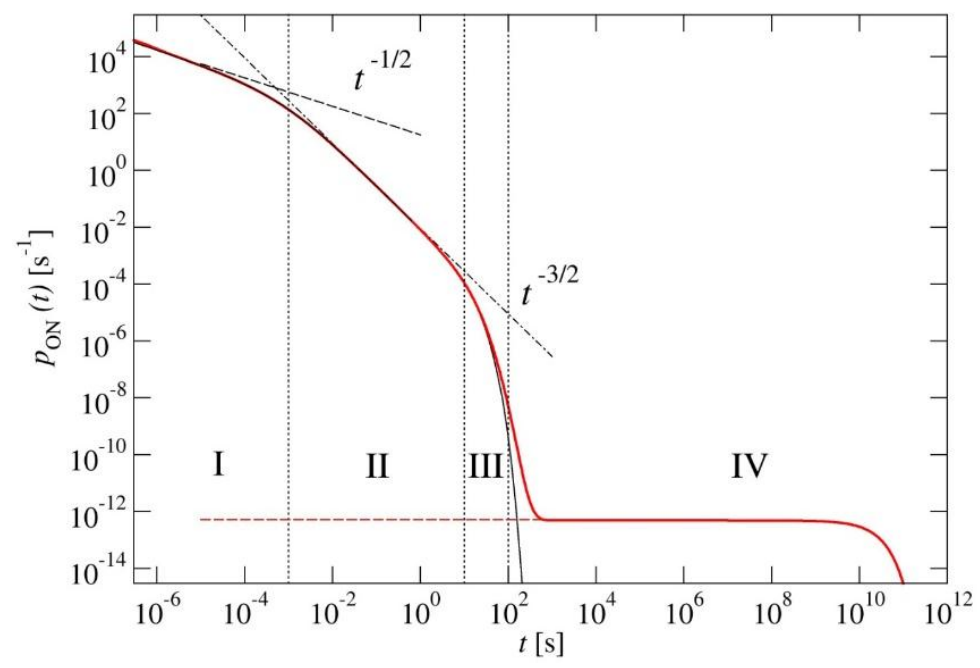

Fig. 1. The ON time distribution function within the DCET model (thick red line), the first interval power law (black dashed line), the second interval power law (black dashed-dotted line), the TangMarcus approximation (thin black line) and the long-time exponential asymptotic (dashed red line).

The study was supported by the Russian Foundation for Basic Research, project 16-02-00713.

\section{References}

1. M. Nirmal, B. O. Dabousi, M. G. Bawendi, J. J. Maklin, J. K. Trautman, T. D. Harris, and L. E. Brus, Nature, 383, 802 (1996)

2. P. A. Frantsuzov, M. Kuno, B. Janko, and R. A. Marcus, Nature Phys., 4, 519 (2008)

3. F. Cichos, C. von Borczyskowski, and M. Orrit, Curr. Opin. Colloid. Interface Sci., 12, 272 (2007)

4. J. A. Cordones and S. R. Leone, Chem. Soc. Rev., 42, 3209 (2013)

5. S. A. Empedocles, D. J. Norris, and M. G. Bawendi, Phys. Rev. Lett., 77, 3873 (1996)

6. S. A. Empedocles and M. G. Bawendi, J. Phys. Chem. B, 103, 1826 (1999)

7. K. T. Shimizu, R. G. Neuhauser, C. A. Leatherdale, S. A. Empedocles, W. K.Woo, and M. G. Bawendi, Phys. Rev. B, 63, 205316 (2001)

8. A. L. Efros and M. Rosen, Phys. Rev. Lett., 78, 1110 (1997)

9. J. Tang and R. A. Marcus, J. Chem. Phys., 123, 054704 (2005)

10. J. Tang and R. A. Marcus, Phys. Rev. Lett., 95, 107401 (2005)

11. Z. Zhu and R. A. Marcus, Phys. Chem. Chem. Phys., 16, 25694 (2014)

12. P. A. Frantsuzov and R. A. Marcus, Phys. Rev. B, 72, 155321 (2005) 